\title{
Cellular Digital Fibroma: A Comprehensive Review of a CD34-Positive Acral Lesion of the Distal Fingers and Toes
}

Philip R. Cohen · Robert S. Alpert - Antoanella Calame

Received: June 5, 2020 / Published online: July 29, 2020

(C) The Author(s) 2020
Digital Features To view digital features for this article go to https://doi.org/10.6084/m9.figshare.12534293.

P. R. Cohen

San Diego Family Dermatology, National City, CA, USA

P. R. Cohen

Touro University California College of Osteopathic Medicine, Vallejo, CA, USA

\section{A. Calame}

Compass Dermatopathology, San Diego, CA, USA

P. R. Cohen $(\triangle)$

10991 Twinleaf Court, San Diego, CA 92131, USA

e-mail: mitehead@gmail.com
$5 \mathrm{~mm}$ or smaller. It frequently occurred on either the dorsal, lateral or ventral side of a digit. Yet, some of the lesions were located on the nail fold of the digit. Cellular digital fibroma shows a prominent cellular proliferation of spindle-shaped fibroblasts, without any atypia or mitoses, that extends from the papillary into the upper reticular dermis; diffuse and strongly positive CD34 staining is present throughout the entire tumor. There is no erosion by the tumor of the bony phalanx. Other acral tumors, such as superficial acral fibromyxoma (which also has diffuse strongly positive CD34 staining) and acquired digital fibrokeratoma (which is either CD34-negative or only focal CD34 positive), are in the clinical and pathologic differential diagnosis of cellular digital fibroma. Conservative complete excision is the treatment of cellular digital fibroma; however, even for tumors that have only been partially removed during biopsy, recurrence has not been observed. In conclusion, cellular digital fibroma is a unique CD34-positive acral lesion of the distal fingers and toes whose diagnosis requires correlation of the clinical morphology and the pathologic features of the tumor.

Keywords: Acquired; Acral; CD34; CD99; Cellular; Digital; Fibrokeratoma; Fibroma; Fibromyxoma; Finger; Periungual; Subungual; Superficial; Toe 


\section{Key Summary Points}

Cellular digital fibroma is a distinctive CD34-positive benign lesion of the distal fingers and toes whose diagnosis requires correlation of the tumor's clinical morphology and the pathologic features in order to exclude other acral tumors in the differential diagnosis such as superficial acral fibromyxoma and acquired digital fibrokeratoma

Cellular digital fibroma presents as a slowgrowing, typically asymptomatic, erythematous or flesh-colored, small (usually less than $5 \mathrm{~mm}$ ), solitary papule that is located either on the dorsal, lateral, or ventral side of a digit or the digit's nail fold

Cellular digital fibroma shows a dense collagenous dermal stroma with a prominent cellular proliferation of spindle-shaped fibroblasts extending from the papillary dermis into the upper reticular dermis; the overlying epidermis shows compact orthokeratosis and acanthosis - with or without an epithelial collarette extending into the dermis and surrounding the tumor

Cellular digital fibroma shows diffuse and strongly positive CD34 staining, positive vimentin staining, and focal positive factor XIIIa staining; they do not stain with epithelial membrane antigen (EMA), S100, desmin, muscle-specific actin, smooth muscle actin, and tryptase

The treatment of cellular digital fibroma is conservative complete excision; however, recurrence has not been observed even after partial tumor removal

\section{INTRODUCTION}

Cellular digital fibroma is a benign fibrous lesion that has been described in 20 individuals. Clinically, it typically appears as an asymptomatic small papule either on the side of the digit or on the nail fold. Pathologically, extending from the papillary dermis into the upper reticular dermis, the lesion shows short intersecting fascicles of spindle-shaped fibroblasts oriented either parallel to the epidermis or in a haphazard manner accompanied by a variable dense dermal collagen, subtle vascularity and minimal to no myxoid component; there is diffuse strongly cluster of differentiation 34 (CD34)-positive staining of the tumor.

The purpose of this paper is to summarize the features of cellular digital fibroma (Tables 1 and 2) [1-6]. This article is based on previously conducted studies and does not contain studies with human participants or animals performed by any of the authors. However, the patient signed a consent providing permission for including the clinical photographs in this article.

\section{DISCUSSION}

\section{History}

In July 2005, a unique subset of digital fibromas that were composed of CD34-postive slender spindle cells was introduced by McNiff et al. from New Haven, Connecticut [3]. In their study, they also referred to a consultation case provided by Dr. Michael B. Morgan from Tampa, Florida that had been published more than 3 years earlier, in February 2002 [4]; they considered this patient's tumor to have pathologic features that were remarkably similar, and possibly identical, to the lesions from the 14 cases that they were describing [3]. McNiff et al. expressed concern regarding the fibroma since 2 of the lesions initially prompted them to consider the diagnosis of dermatofibrosarcoma protuberans [3]. The investigators designated these benign acral tumors as cellular digital fibromas [3]. 
Table 1 Characteristics of men with CD34-positive cellular digital fibroma

\begin{tabular}{|c|c|c|c|c|c|c|c|c|c|c|c|}
\hline $\mathrm{C}$ & $\operatorname{Age}^{a}(y r)$ & Dur (mo) & Pain & $\operatorname{Size}^{\mathbf{b}}(\mathrm{mm})$ & Site $^{\mathrm{c}}$ & CD99 & EMA & Factor XIIIa & $S 100$ & $\operatorname{Recur}^{\mathrm{d}}(\mathrm{mo})$ & Ref \\
\hline 1 & 27 & 12 & Slt & 10 & FR2 & NP & NP & NP & NP & NS & 1 \\
\hline 2 & 38 & 6 & None & 5 & HR2 & - & NP & NP & - & No (18) & 2 \\
\hline 3 & 42 & NS & NS & NS & HR3 & NP & e & $+^{\mathrm{f}}$ & e & $\mathrm{g}$ & $3 \mathrm{C} 8$ \\
\hline 4 & 47 & NS & NS & NS & HR1 & NP & e & $+{ }^{\mathrm{f}}$ & e & $\mathrm{g}$ & $3 \mathrm{C} 7$ \\
\hline 5 & 51 & NS & NS & NS & $\mathrm{HL}^{\mathrm{h}}$ & $\mathrm{NP}$ & e & $+^{\mathrm{f}}$ & e & $\mathrm{g}$ & $3 C_{5}$ \\
\hline 6 & 53 & 2 & NS & 5 & $\mathrm{FR}^{\mathrm{i}}$ & NP & NP & - & - & NS & 4 \\
\hline 7 & 55 & 24 & NS & NS & HR2 & $+^{\mathrm{j}}$ & - & NP & - & No (18) & 5 \\
\hline 8 & 56 & NS & NS & NS & $\mathrm{HR}^{\mathrm{k}}$ & NP & e & $+{ }^{\mathrm{f}}$ & e & $\mathrm{g}$ & $3 \mathrm{C} 4$ \\
\hline 9 & 60 & 10 & None & 5 & HR2 & $\mathrm{NP}$ & - & - & - & No (18) & 6 \\
\hline 10 & 61 & 18 & None & 5 & FL1 & NP & - & $+{ }^{1}$ & - & No (5) & $\mathrm{CR}$ \\
\hline 11 & 67 & NS & NS & NS & HR3 & NP & e & $+^{\mathrm{f}}$ & e & $\mathrm{g}$ & $3 \mathrm{C} 1$ \\
\hline 12 & 82 & NS & NS & NS & FL1 & $\mathrm{NP}$ & e & $+{ }^{\mathrm{f}}$ & e & $\mathrm{g}$ & $3 \mathrm{C} 12$ \\
\hline
\end{tabular}

$C$ case, $C D$ cluster of differentiation, $C R$ current report, Dur duration that cellular digital fibroma was present prior to diagnosis, $E M A$ epithelial membrane antigen, $F$ foot, $H$ hand, $L$ left, $m m$ millimeters, mo months, $N P$ not performed, $N S$ not stated, $R$ right, Recur recurrence, Ref reference, Slt slightly painful on palpation, $y r$ years, - negative, + positive

${ }^{a}$ The age of the patient when the cellular digital fibroma was diagnosed

b The size is the measurement of the greatest dimension of the cellular digital fibroma

$c$ The site of the cellular digital fibroma is described by either the hand $(\mathrm{H})$ or the foot $(\mathrm{F})$, the left $(\mathrm{L})$ or right $(\mathrm{R})$ side, and the specific digit: 1 , thumb or great (or first) toe; 2 , index finger or second toe; 3 , middle finger or third toe; 4 , ring finger or fourth toe; 5, baby (or pinky) finger or little (or fifth) toe

$\mathrm{d}$ The number of months after diagnosis and treatment that follow up examination was performed and no clinical recurrence was observed

e The study by McNiff et al. [3] included six men and eight women; five of the 14 cases were evaluated for EMA and S100 and were found to be negative

${ }^{\mathrm{f}}$ Factor X111a labeled only scattered spindle or stellate cells

g The study by McNiff et al. [3] included 6 men and 8 women. The two patients who returned for clinical follow up had no evidence of disease; the other 12 patients did not return for follow-up examination

h The exact site of the cellular digital fibroma was not described

${ }^{i}$ The cellular digital fibroma was located on the dorsum of the foot

${ }^{j}$ Focal positivity for CD99 was observed

${ }^{k}$ The cellular digital fibroma was located on the palm

${ }^{1}$ Only a few cells were observed to show positive staining for factor XIIIa

Nearly one and a half years later, in November 2006, Guitart et al. confirmed that the lesion could easily be misdiagnosed as a dermatofibrosarcoma protuberans [7]. However, this group of researchers questioned whether McNiff et al.'s cellular digital fibroma was different from an essentially identical case that they had diagnosed as a superficial acral fibromyxoma in the past. They concluded not only that awareness of this entity was important, but also that it would be necessary to determine if indeed the cellular digital fibroma and the superficial acral fibromyxoma were different conditions [7]. 
Table 2 Characteristics of women with CD34-positive cellular digital fibroma

\begin{tabular}{llll}
\hline Case & Age $^{\mathbf{a}}$ & Site $^{\mathbf{b}}$ & References \\
\hline 1 & 33 years & Foot, Left: 1 & 3, case 6 \\
2 & 37 years & Foot, Left: 2 & 3, case 2 \\
3 & 40 years & Hand, Right: 4 & 3, case 11 \\
4 & 45 years & Foot, Right: 2 & 3, case 9 \\
5 & 50 years & Foot, Left & 3, case 10 \\
6 & 53 years & Hand, Right: 2 & 3, case 13 \\
7 & 77 years & Hand, Left: 1 & 3, case 3 \\
8 & 83 years & Hand, Right: 3 & 3, case 14 \\
\hline
\end{tabular}

The study by McNiff et al. [3] included six men and eight women. All of the cases showed Factor XIIIa to label only scattered spindle or stellate cells. Five of the 14 cases were evaluated for EMA and S100 and were found to be negative. The two patients who returned for clinical follow up had no evidence of disease; the other 12 patients did not return for follow-up examination

${ }^{a}$ The age of the patient when the cellular digital fibroma was diagnosed

b The site of the cellular digital fibroma is described by either the hand or the foot, the left or right side, and the specific digit: 1 , thumb or great (or first) toe; 2 , index finger or second toe; 3 , middle finger or third toe; 4 , ring finger or fourth toe; 5, baby (or pinky) finger or little (or fifth) toe

c The exact site of the cellular digital fibroma was not described

In their reply to Guitart et al., McNiff et al. acknowledged the similarities between the two tumors-specifically that both were acral lesions defined by delicate CD34-positive spindled cells in a variable stroma [8]. However, McNiff et al. emphasized important differences between the two tumors [8]. In contrast to the cellular digital fibroma, the superficial acral fibromyxoma tended to involve the nail matrix, had a distinctly more myxoid stroma with less cellularity and increased vascularity, and often stained positive with epithelial membrane antigen [8].

Since McNiff et al.'s seminal paper [3], we have been able to identify four additional case reports of cellular digital fibroma $[1,2,5,6]$. The origins of the reports were world-wide; they were published in journals from Argentina [1], Korea [2], Spain [5], and the United States of America [6]. In addition, in this paper, we contribute one more case of cellular digital fibroma; hence to the best of our knowledge, this brings the total number of patients in whom a cellular digital fibroma has been described to 20 individuals.

\section{Nomenclature}

In 2002, four dermatopathologists offered their opinion on a spindle cell CD34 + dermal proliferation provided by Dr. Morgan. The lesion was a solitary $5 \mathrm{~mm}$ dome-shaped papule on the dorsum foot of a 53-year-old man. There had been no previous trauma or surgery to the site and the lesion developed over a 2 month period. The spindle and stellate cells of the papule stained with antibody to vimentin; however, they did not stain with antibody to actin, desmin, factor XIIIa, S-100 or tryptase [4].

Dr. Pitha suggested that the neoplasm be given a generic designation: dermal myxoid fibroma with the descriptor of CD34 positivity. Dr. Smoller (who discussed the case with Dr. Horn) favored the lesion to represent a benign neoplasm of the follicle-associated mesenchyme. Dr. Somach believed that the tumor was a benign, yet undefined, proliferation of periadnexal CD34 + dendritic cells associated with a subtle induction of epithelial strands. And Dr. McCalmont considered the tumor to be a benign dermal spindle cell proliferation with avid CD34 positive dendritic cell expression of the type conspicuous in the adventitial dermis. Other diagnoses that were submitted by the group of consultants included dermatofibrosarcoma protuberans, neurofollicular hamartoma, neurothekeoma and sclerotic fibroma [4].

Three years later, in 2005, McNiff et al. decided that the tumor submitted for consultation by Dr. Morgan in 2002 was similar, if not identical, to the 14 cases they presented as cellular digital fibroma [3]. In 2006, the group also offered the name 'nonmyxoid fibromyxoma' [8]; however, this term did not gain acceptance. Similarly, in 2019, Mancuso et al. suggested that 
a cellular digital fibroma was a mucin-poor superficial acral fibromyxoma [9].

However, since McNiff et al.'s original paper [3], the 5 subsequently published cases (including this report) of this entity have referred to the tumor as a cellular digital fibroma.

\section{Epidemiology}

To the best of our knowledge (and including the individual presented in the legends of Figs. 1, 2, 3 and 4 in this manuscript), cellular digital fibroma has been described in 20 patients: 12 men and 8 women (Tables 1 and 2) [1-6]. Hence, the ratio of men to women was 1.5:1.0.

The onset age of cellular digital fibroma ranged in men from 27 to 82 years old (median, 54 years old; mean, 53 years old). In women, the onset age ranged from 33 to 83 years old (median, 48 years old; mean, 52 years old). Overall, the age ranged from 27 to 83 years old (median, 52 years; mean, 53 years).

\section{Duration}

The duration that the cellular digital fibroma was present prior to diagnosis was described in 6 men. It ranged from 2 months to 2 years. The median duration was 11 months and the mean duration was 1 year.

\section{Symptoms}

A specific comments stating that the cellular digital fibroma was asymptomatic was only mentioned for the patient in this paper and 2 other men $[2,6]$. A larger cellular digital fibroma on the proximal nail fold the right second toe of a 27-old-man was slightly painful on palpation [1]. The presence of symptoms was not described for any of the other patients with a cellular digital fibroma. This is in contrast to patients with superficial acral fibromyxoma in whom from $10 \%(4 / 41)$ [10] to $31 \%(5 / 16)$ [11] to $41 \%$ $(19 / 46)$ [12] presented with painful tumors $[13,14]$.
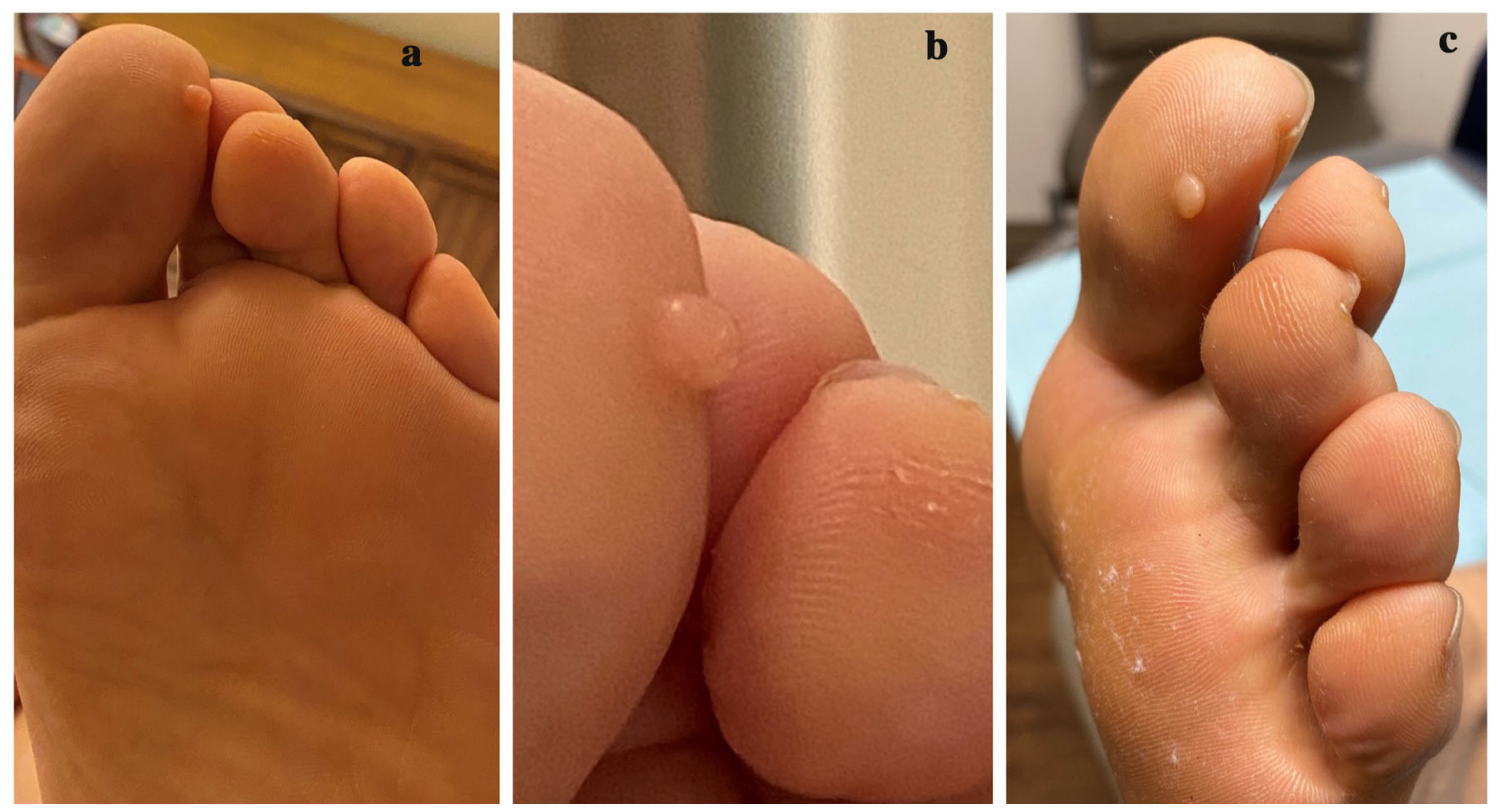

Fig. 1 Clinical presentation of a cellular digital fibroma on the left great toe. Distant $(\mathbf{a}, \mathbf{c})$ and closer $(\mathbf{b})$, plantar (a, b) and lateral (c) views of a new asymptomatic fleshcolored $5 \mathrm{~mm}$ papule on the lateral side of the left great toe of a 61-year-old man. The lesion had been present for 18 month and had been enlarging slowly until it stopped growing 4 months ago. There had been no trauma to the site 


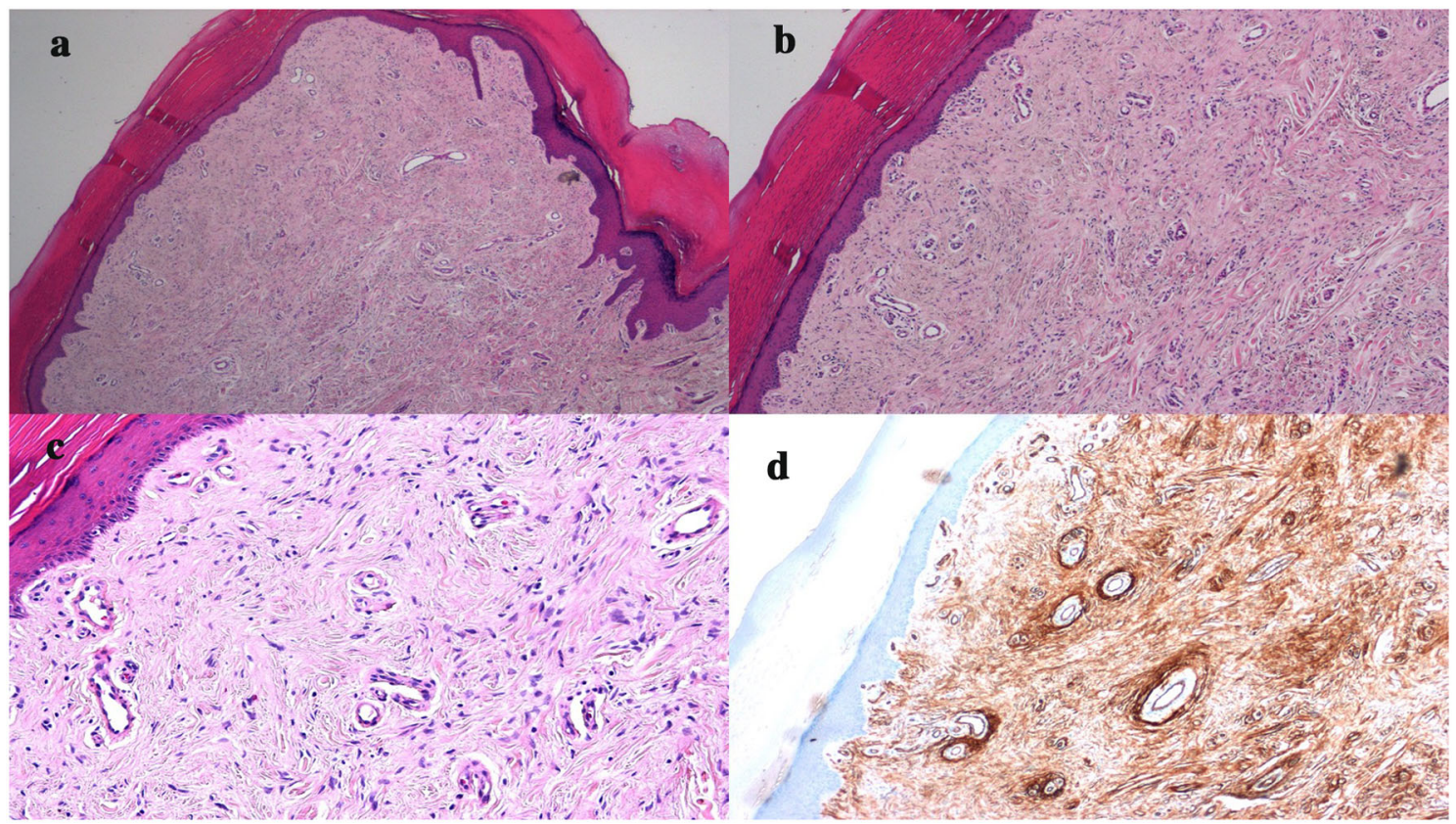

Fig. 2 Microscopic features of a cellular digital fibroma that was present on the left great toe of a 61-year-old man. Distant (a) and closer (b-d) views of the cellular digital fibroma show characteristic pathologic features. The hematoxylin and eosin $(\mathbf{a}-\mathbf{c})$ stained sections show compact orthokeratosis and acanthosis of the epidermis.

Cellular digital fibroma is usually not preceded by trauma to the area $[4,5]$. It is a slow growing lesion. It starts as a small papule and gradually increases in size. The cellular digital fibroma on the toe of the patient in this paper stopped enlarging after developing to its full size.

\section{Location}

The location of the cellular digital fibroma was available for all of the 20 patients (Tables 1,2 and 3) [1-6]. It characteristically occurred either on the hands (12 patients) or the feet (8 patients). The cellular digital fibroma was located on hand of 8 men (with 7 on right and 1 on the left) and the foot of 4 men (with 2 on the right and 2 on the left). The cellular digital fibroma was located on hand of 4 women (with 3 on right and 1 on the left) and the foot of 4 women (with 3 on the left and 1 on the right).
In the predominantly collagenous dermis, there is a tumor composed of spindle-shaped fibroblasts and minimal myxoid stroma. Neither cellular atypia nor mast cells are noted. The CD34 stained section shows diffuse and strong staining of the entire dermal tumor (hematoxylin and eosin: $\mathbf{a} \times 2 ; \mathbf{b}, \times 4 ; \mathbf{c} \times 10 ; \mathrm{CD} 34: \mathbf{d}, \times 10)$

Cellular digital fibroma usually appears on the digits [3]. Often, it was located on the dorsal [6], lateral (similar to the patient in this report), or ventral [2] side of the digit. However, some of the cellular digital fibroma developed in the periungual nail fold $[1,5]$.

The cellular digital fibroma on the hands most frequently occurred on the thumb (3 patients), index finger (3 patients) and middle finger (3 patients); 1 patient had her lesion on the ring finger. The foot lesions most frequently occurred either on the great toe (3 patients) or the second toe (3 patients). The location of the cellular digital fibroma on a 50-year-old woman's left foot and another on a 51-year-old man's left hand were not described [3].

Ectopic cellular digital fibroma refers to a lesion that did not occur on the digit. A cellular digital fibroma was located on the right palm of a 56-year-old man and another cellular digital fibroma developed on the dorsum of the right foot of a 53-year-old man $[3,4]$. Therefore, the 


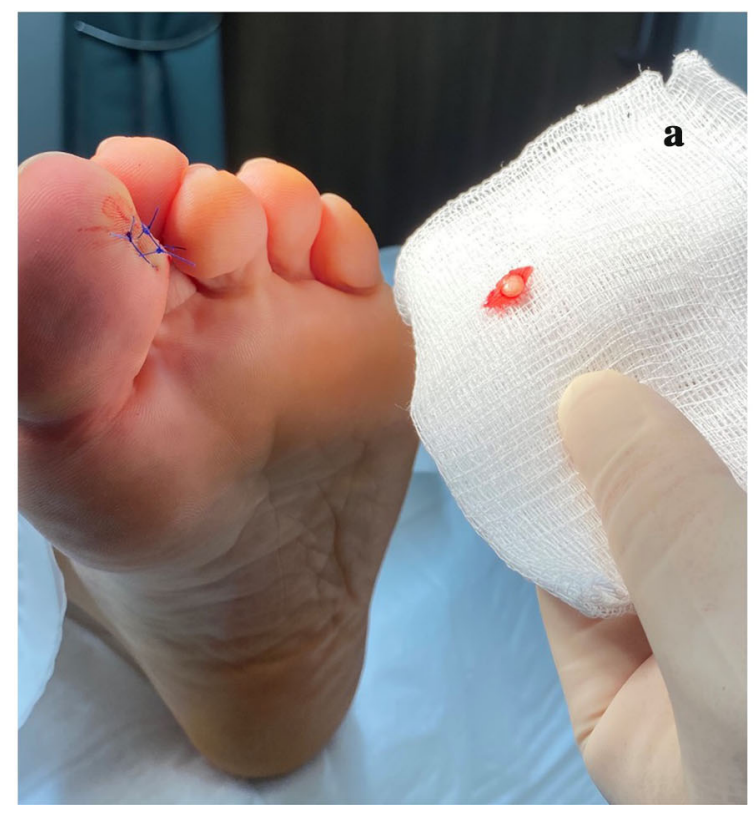

Fig. 3 Excision specimen of cellular digital fibroma that had been present on the left great toe of a 61-year-old man. Distant (a) and closer (b) views of the gross specimen (a) and the wound repair (a, b) following an elliptical

observed incidence of ectopic cellular digital fibroma is as low as $10 \%$ ( 2 of 20 lesions) to as high as $20 \%$ ( 4 of 20 lesions if both the foot and hand location that were not described-in the 50-year-old woman and the 51-year-old man, respectively-were actually not on a digit).

\section{Clinical Presentation}

Cellular digital fibroma is a solitary tumor. The size of the cellular digital fibroma was described for $5 \mathrm{men}$; it ranged from $5 \mathrm{~mm}$ to $1 \mathrm{~cm}$ in greatest dimension. The median size was $5 \mathrm{~mm}$ and the mean size was $6 \mathrm{~mm}$.

The color of the cellular digital fibroma was pink in a 60-year-old man with a wart-like small dome-shaped papule on his right index finger [6]. Another report shares that the lesion appeared as an erythematous fixed protruding nodule on the ventral surface of the proximal phalanx of a 38-year-old man's right index finger [2]. Clinical images show the cellular digital fibroma presenting as a red nodule on the right second toe proximal nail fold of a 27 -year-old man [1] and the right index finger lateral nail

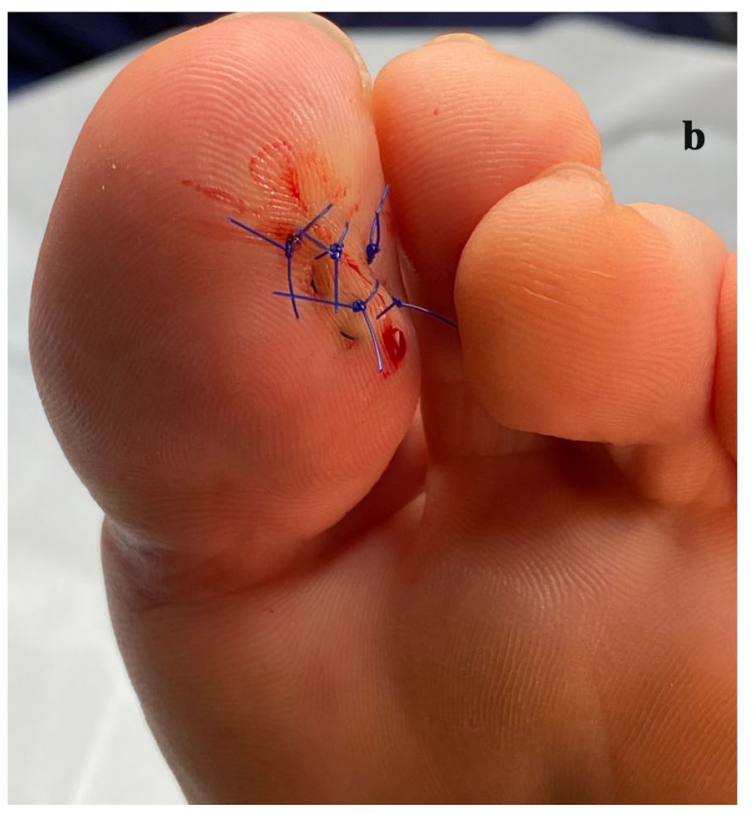

excisional biopsy of the cellular digital fibroma. The wound was closed with polypropylene (prolene) suture using two horizontal mattresses and three interrupted stitches

fold of a 55-year-old man [5]. The cellular digital fibroma was flesh-colored on the left great toe in the 61-year-old man described in this paper (Fig. 1).

In the study by McNiff et al. [3], all of the lesions presented as $<5 \mathrm{~mm}$, benign-appearing papules. Most of lesions morphologically resembled warts. Indeed, the submitted diagnosis from the clinician was either wart or fibroma [3]. The clinical appearance of the cellular digital fibroma in the patient in this report was an elongated papule; the differential diagnoses included an acrochordon (skin tag) and an acquired digital fibrokeratoma.

\section{Pathology}

\section{Hematoxylin and Eosin Staining}

The pathologic changes of cellular digital fibroma uniformly usually show hyperkeratosis-that may be compact-without the retention of cell nuclei of the stratum corneum (orthokeratosis) and thickening of the epidermis (acanthosis) (Fig. 2) [3, 5, 6]. An 'epidermal collarette'-created by elongation of the 


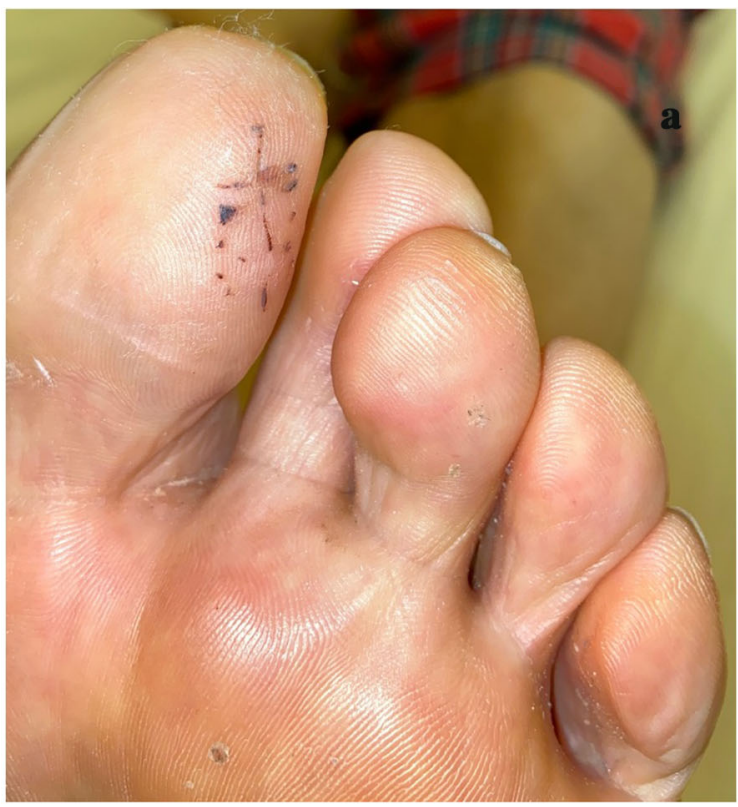

Fig. 4 Postoperative examination and subsequent follow up visit after complete removal of cellular digital fibroma from the left great toe of a 61-year-old man. The excision site of the cellular digital fibroma has completely healed when the sutures were removed at the follow up visit 2

epidermal rete ridges at the periphery of the lesion-may extends into the underlying dermis and surround the dermal fibrous component of the cellular digital fibroma $[2,3,6]$.

In the dermis, there is a proliferation of fibroblasts that are uniformly slender. The spindle-shaped cells for short interweaving fascicles that extend from the papillary dermis into the upper reticular dermis. The fascicles are either oriented parallel to the epidermis or in a haphazard (storiform) manner or both (Fig. 2) $[3,6]$.

The dermal stroma is characterized by variable dense collagen; vascularity is subtle. A myxoid dermal component is also often present, yet minimal. Indeed, in cellular digital fibroma, the collagenous component of the dermis is typically more prominent than the myxoid stroma $[3,6]$.

In cellular digital fibroma, there is no pleomorphism or atypia of the tumor fibroblasts. Mitoses of the spindle-shaped cells' nuclei are absent. Mast cells in the dermis are few or none $[3,6,8]$.

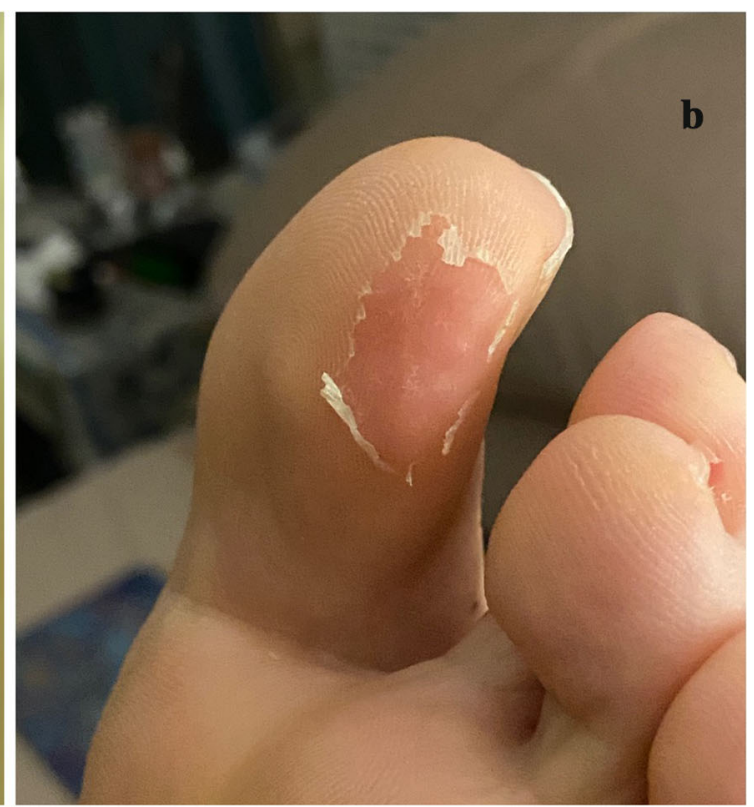

weeks after the excisional biopsy (a). There is superficial desquamation of the epidermis surrounding the excision site and the surgical scar is faintly visible 3 weeks after the procedure was performed $(\mathbf{b})$. There has been no recurrence of the cellular digital fibroma at 5 months follow up

\section{Mucin Staining}

Alcian blue stain can be used to demonstrate the myxoid component in the dermis of the cellular digital fibroma [2]. Similar findings would be anticipated with other stains that highlight mucin such as colloidal iron or mucicarmine or periodic acid-Schiff.

\section{Immunoperoxidase Staining}

Cluster of Differentiation 34 (CD34) CD34 is the human hematopoietic progenitor cell antigen $[15,16]$. Cellular digital fibroma characteristically demonstrates not only diffuse but also strongly positive staining with CD34 (Fig. 2) $[1-6,8]$. This is a pathognomonic feature of the tumor. However, superficial acral fibromyxoma-a tumor in the clinical and pathological differential diagnosis of cellular digital fibroma-also shows similar CD34 positivity [11].

Indeed, the prominent cellularity and strong CD34 positivity of the cellular digital fibroma in McNiff et al.'s study caused the investigators to be sufficiently concerned about the possibility of dermatofibrosarcoma protuberans for 2 of the 
Table 3 Location of cellular digital fibromas

\begin{tabular}{|c|c|c|c|c|c|c|c|c|}
\hline \multicolumn{2}{|c|}{ Location $^{\mathrm{a}}$} & \multicolumn{2}{|l|}{ Men } & \multicolumn{2}{|c|}{ Women } & \multicolumn{2}{|c|}{ Total } & \multirow[t]{2}{*}{ Total } \\
\hline Site & Digit & Left & Right & $\overline{\text { Left }}$ & Right & $\overline{\text { Left }}$ & $\overline{\text { Right }}$ & \\
\hline Foot & 1 & 2 & - & 1 & - & 3 & - & 3 \\
\hline Foot & 2 & - & 1 & 1 & 1 & 1 & 2 & 3 \\
\hline Foot & 3 & - & - & - & - & - & - & - \\
\hline Foot & 4 & - & - & - & - & - & - & - \\
\hline Foot & 5 & - & - & - & - & - & - & - \\
\hline Foot & Other & - & $1^{\mathrm{b}}$ & $1^{\mathrm{c}}$ & - & 1 & 1 & 2 \\
\hline Total & & 2 & 2 & 3 & 1 & 5 & 3 & 8 \\
\hline Hand & 1 & - & 2 & 1 & - & 1 & 2 & 3 \\
\hline Hand & 2 & - & 2 & - & 1 & - & 3 & 3 \\
\hline Hand & 3 & - & 2 & - & 1 & - & 3 & 3 \\
\hline Hand & 4 & - & - & - & 1 & - & 1 & 1 \\
\hline Hand & 5 & - & - & - & - & - & - & - \\
\hline Hand & Other & $1^{\mathrm{c}}$ & $1^{\mathrm{d}}$ & - & - & 1 & 1 & 2 \\
\hline Total & & 1 & 7 & 1 & 3 & 2 & 10 & 12 \\
\hline Total & & 3 & 9 & 4 & 4 & 7 & 13 & 20 \\
\hline
\end{tabular}

a The location of the cellular digital fibroma is described by the site (either the hand or the foot) and the specific digit: 1 , thumb or great (or first) toe; 2, index finger or second toe; 3, middle finger or third toe; 4, ring finger or fourth toe; 5 , baby (or pinky) finger or little (or fifth) toe

b The cellular digital fibroma was located on the dorsum of the foot

c The exact site of the cellular digital fibroma was not described

d The cellular digital fibroma was located on the palm

14 cases [3]. They contacted the clinicians for these 2 lesions; both clinicians confirmed that the lesions (small dome-shaped papules) were not clinically consistent with the suspected diagnosis. In addition, 1 of the clinician re-excised the lesion [3].

Since the original descriptions of cellular digital fibroma in 2005 [3] and superficial acral fibromyxoma in 2001 [11], researchers have observed another stain that can be used to differential dermatofibrosarcoma protuberans: apolipoprotein D. Apolipoprotein D is a 33-kilodalton glycoprotein component of highdensity lipoprotein. It is highly expressed in dermatofibrosarcoma protuberans; however, researchers did not observe any expression of apolipoprotein D in 4 superficial acral fibromyxoma that they evaluated [17].

Cluster of Differentiation 99 (CD99) CD99 antigen, also known as MIC2 or single-chain type-1 glycoprotein, is a highly O-glycosylated transmembrane protein; it is encoded by the CD99 gene which is also referred to as the pseudoautosomal gene MIC2 in humans [18]. CD99 staining has been evaluated in the cellular digital fibroma from 2 patients. Focal positivity for CD99 was noted on the right index finger lesion of a 55-year-old man [5]. However, another cellular digital fibroma on right index finger of a 38-year-old man was CD99-negative [2]. 
Vimentin Vimentin is a type III intermediate filament protein that is expressed by mesenchymal cells [19]. Cellular digital fibroma is vimentin positive. The dermal tumor cells from both patients whose cellular digital fibroma was evaluated-a 27-year-old man with a right second toe lesion and 38-year-old man with a right index finger lesion-expressed vimentin $[1,2]$.

Factor XIIIa Factor XIIIa is a coagulation factor produced in blood and intracellularly; in normal skin it is expressed by dermal dendrocytes [20-22]. Cellular digital fibroma showed sparse of no factor XIIIa expression. Two patients-a 53-year-old man with a cellular digital fibroma on his right foot and a 60-yearold man with a cellular digital fibroma on his right index finger-had lesions that were factor XIIIa-negative $[4,6]$. The patient in this reports left great toe lesion only had a few cells in the dermis that stained positive for factor XIIIa. In McNiff et al.'s study, factor XIIIa labeled only scattered spindle or stellate cells in the dermis [3].

\section{Epithelial Membrane Antigen Epithelial} membrane antigen (EMA), also referred to as MUC1, is a transmembrane mucin or glycoprotein [23]. None of the cellular digital fibroma ( 0 of 8$)$, including our patient's lesion, expressed EMA $[3,5,6]$.

S100 In normal skin, melanocytes and Langerhans cells in the epidermis and Schwann cells in the dermis express $\mathrm{S} 100$ protein. Benign (nevi, neuromas and schwannomas) and malignant (melanoma and peripheral nerve sheath tumors) melanocytic and neural tumors also S100-positive [24, 25]. None of the cellular digital fibroma ( 0 of 10$)$, including our patient's lesion, expressed S100 protein [2-6].

\section{Diagnosis}

The diagnosis of cellular digital fibroma may be suspected based upon the clinical evaluation of new asymptomatic tumor on either the lateral or dorsal or ventral aspect of the digit or, less commonly, the proximal nail fold. If the tumor is painful or subungual, the more likely diagnosis is a superficial acral fibromyxoma. Acquired digital fibrokeratoma has similar morphologic features to a cellular digital fibroma; therefore differentiating these lesions requires microscopic evaluation of the tumor.

A biopsy-either partial or excisional-is necessary to evaluate the digital lesion. Evaluation of tissue section stained with hematoxylin and eosin and CD34 should be performed. A stain that demonstrates the myxoid component, such as alcian blue, may be helpful. In addition, other immunoperoxidase stainssuch as EMA, factor XIIIa, S100, and vimentinmay be useful. Other investigators have also evaluated the tumors with staining for desmin, muscle-specific actin and smooth muscle actin.

In summary, a correlation of the clinical presentation and the pathology findings is recommended to establish a diagnosis of cellular digital fibroma. In contrast to cellular digital fibroma, a subungual tumor with marked myxoid stroma that stains strongly positive with CD34 is a superficial acral fibromyxoma and a lateral digit tumor with fibroblasts oriented perpendicular to the overlying epidermis and staining sparsely (or not staining) with CD34 is an acquired digital fibrokeratoma. The cellular digital fibroma is a tumor on either the digit or nail fold that is more cellular than myxoid and stains strongly positive with CD34.

\section{Differential Diagnosis}

\section{Clinical}

The principal tumors that morphologically mimic a cellular digital fibroma are a superficial acral fibromyxoma and an acquired digital fibrokeratoma (Table 4) $[1-5,7-9,11,12$, 26-33]. Several other conditions are also in the clinical differential diagnosis. Some of these include acquired reactive digital fibroma, digital fibroma, periungual fibroma (referred to as a Koenen tumor located under the nail fold and associated with tuberous sclerosis when multiple lesions are present), and supernumerary digit and verruca vulgaris [29, 34, 35]. 
Table 4 Comparison of features of cellular digital fibroma, superficial acral fibromyxoma and acquired digital fibrokeratoma

\begin{tabular}{clll}
\hline Feature & Cellular digital fibroma & $\begin{array}{l}\text { Superficial acral } \\
\text { fibromyxoma }\end{array}$ & Acquired digital fibrokeratoma \\
\hline Morphology & Papule (dome-shaped) & Nodule & Papule (filiform) \\
Clinical & Side of digit & Subungual & Side of digit \\
location & Periungual nail fold & Periungual nail fold & \\
Bony erosion & Never & Occasionally & Never \\
Pathology & Dermis & Dermis; can extend into & Dermis \\
location & & subcutaneous fat & \\
H\&E & Spindled fibroblasts & Spindled fibroblasts & Fibroblasts and dense collagen vertically oriented to \\
& Lack stellate cells & Stellate cells in dermis & the overlying epidermis \\
& Slight myxoid stroma & Marked myxoid stroma & \\
& Variable dense collagen & Sparse dense collagen & \\
CD34 & Diffuse and strongly & Diffuse and strongly & Focally positive or negative \\
& positive staining & positive staining & \\
CD99 & Positive or negative & Usually positive & ?b \\
Vimentin & Positive & Positive & Positive \\
Factor XIIIa & Focally positive & Focally positive & Focally positive \\
EMA & Negative & Usually positive & Negative \\
S100 & Negative & Negative & Negative \\
\hline
\end{tabular}

$C D$ cluster of differentiation, $H \& E$ hematoxylin and eosin, $E M A$ epithelial membrane antigen, ? unknown

These comments for the features in each of the lesion categories are the findings that are usually observed

a CD99 has only been evaluated in two cellular digital fibromas. Focal positivity of CD99 was observed in one man's lesion [5] and another man's tumor was negative for CD99 [2]

$\mathrm{b}$ To the best of our knowledge, evaluation of CD99 staining of acquired digit fibrokeratoma has not been described

\section{Superficial acral fibromyxoma Superficial} acral fibromyxoma are slow growing myxoid tumors that preferentially affect the subungual and periungual areas of the digits. Similar to cellular digital fibroma, ectopic superficial acral fibromyxoma-not located on the digits-have been described; the incidence of ectopic superficial acral fibromyxoma ranges from $2.5 \%$ (1/ $41)[10]$ to $8 \%(3 / 37)$ [11] to $9 \%(11 / 124)$ [12] to $12.5 \%(4 / 32)$ [36]. In contrast to cellular digital fibroma that do not have a prior history of tumor site-associated trauma and are almost universally asymptomatic, superficial acral fibromyxoma may be preceded by trauma to the location in $19 \%$ (7 of 37 ) [11] to $25 \%$ (14 of 55)
[12] of cases and up to $41 \%(19 / 46)$ of the lesions are painful [12]. Also, in contrast to cellular digital fibroma that do not affect the underlying bone and do not recur, superficial acral fibromyxoma is not only associated with invasion of the digit's bone in 25\% (1 of 4) [37] to $36 \%$ (9 of 25) [12] of cases, but also with recurrence after incomplete removal ranging from $21 \%$ (3 of 14) [36] to $42 \%$ (5 of 12) [10] of cases.

Acquired digital fibrokeratoma Acquired digital fibrokeratoma is almost always a single lesion on either a finger or a toes $[9,29,38]$. The morphology of acquired digital fibrokeratoma- 
a small, often less than $5 \mathrm{~mm}$, solitary, domeshaped, flesh-colored papule-can be clinically similar to that of a cellular digital fibroma, such as the lesion observed in the 61-year-old man in this report. However, giant acquired digital fibrokeratoma and ectopic acquired digital fibrokeratoma at other sites have been observed [30, 31].

\section{Pathological}

The pathologic differential diagnosis of a cellular digital fibroma also includes superficial acral fibromyxoma and acquired digital fibrokeratoma (Table 4) [1-5, 7-9, 11, 12, 26-33]. However, it also includes but also other benign neoplasms and malignant tumors. Some of the benign neoplasms are acquired reactive digital fibroma, angiomyxoma (superficial), dermatofibroma, infantile digital fibromatosis, neurofibroma (myxoid), and perineuroma (sclerosing) [1-4, 6, 11, 26, 27, 34]. Some of the malignant tumors include dermatofibrosarcoma protuberans (myxoid or superficial biopsy of an early lesion), myxofibrosarcoma (low-grade), $[1,2,5,6,26,27]$. In addition to dermatofibrosarcoma protuberans, the pathological differential diagnosis also includes other CD34positive cutaneous tumors: eruptive CD34-positive fibromas, fibrous papules of the face, lipoma (spindle cell), neuroma, nerve sheath tumors, pleomorphic fibroma, sarcomas (acral myxoinflammatory fibroblastic and epithelioid), sclerotic fibroma, solitary fibrous tumor and vascular lesions (angiosarcoma and Kaposi sarcoma) [1, 3, 11, 26, 27]

Superficial Acral Fibromyxoma Superficial acral fibromyxoma, similar to cellular digital fibroma, often has epidermal changes of compact orthokeratosis and acanthosis; ulceration may also be present. Also similar to cellular digital fibroma, the dermal tumor originates in the papillary dermis and extends into the reticular dermis. However, the superficial acral fibromyxoma lesion may fill the dermis and be present in the subcutaneous fat [11].

In contrast to cellular digital fibroma in which the dermal stroma is predominantly dense collagen, the dermal stroma of a superficial acral fibromyxoma is markedly myxoid,
Yet, the extent of myxoid stroma described is variable. Indeed, in Fetsch et al.'s [11] original description of superficial acral fibromyxoma, the investigators categorized the 37 tumors as having a dermal matrix that was predominantly myxoid (19 cases), myxocollagenous (11 cases) or predominantly collagenous (7 cases).

In contrast to cellular digital fibroma that usually only have spindled fibroblasts, both spindled-shaped and stellate-shaped fibroblastlike cells are present in the dermis of superficial acral fibromyxoma. Also in contrast to cellular digital fibroma, superficial acral fibromyxoma may have mast cells and multinucleated giant cells. Not only cytologic atypia and pleomorphism, but also rare mitoses may be present in superficial acral fibromyxoma; these finding are not present in cellular digital fibroma $[26,28]$.

Although there are occasional exceptions, superficial acral fibromyxoma has a characteristic immunohistochemical profile. Superficial acral fibromyxoma is a CD34-positive tumor; staining is diffuse and strong. Similarly, the tumor typically demonstrates positive staining with vimentin and frequently with CD99; often, the tumor also expresses EMA. Recently, positive staining of superficial acral fibromyxoma with cluster of differentiation 10 (CD10) [39] and nestin [37] has been observed. Factor XIIIa only shows focally positive staining. Superficial acral fibromyxoma do not stain with desmin, HMB45, keratins, muscle-specific actin, smooth muscle actin and S100 [10, 11, 36].

Although some researchers-based on the similarity of some of the pathologic features of superficial acral fibromyxoma and cellular digital fibroma-consider cellular digital fibroma to be a variant of superficial acral fibromyxoma $[7,10,36]$, it is important for the pathologist to correlate the microscopic findings and the clinical morphology of the tumor when establishing the diagnosis of a CD34-positive acral lesion of the distal fingers and toes. In contrast to superficial acral fibromyxoma that are typically subungual or develop in the periungual nail fold, tumors on the dorsal, lateral or ventral side of the digit are more likely to be cellular digital fibroma; however, similar to superficial acral fibromyxoma, cellular digital fibroma can occur in the proximal or lateral nail fold. 
Superficial acral fibromyxoma usually tend to have a dermal stroma that is predominantly myxoid and may extend into the subcutaneous fat; in contrast, cellular digital fibroma has a dense collagenous stroma and is typically restricted to the upper reticular dermis.

Both cellular digital fibroma and superficial acral fibromyxoma demonstrate diffuse and strong staining with vimentin and CD34. However, CD99, which is diffusely and strongly expressed by superficial acral fibromyxoma has not been evaluated sufficiently in cellular digital fibroma-the 2 tumors studied were either CD99-negative [2] or only demonstrated focal positive staining [5]. Similarly, CD10 and nestin-which have been shown to stain superficial acral fibromyxoma-have not been studied in cellular digital fibroma. EMA may stain superficial acral fibromyxoma and-to date-has not been shown to stain cellular digital fibroma; therefore, a CD34-positive digit tumor that expresses EMA is more likely to be a superficial acral fibromyxoma than a cellular digital fibroma. Both cellular digital fibroma and superficial acral fibromyxoma can show focal positivity after staining with factor XIIIa and are S100-negative; hence, although these stains may be helpful to exclude other spindle cell tumors, they are not useful for differentiating between cellular digital fibroma and superficial acral fibromyxoma.

Acquired Digital Fibrokeratoma Kint et al. [32] published the results of a retrospective study of 50 cases of acquired digital fibrokeratoma in 1985. They discovered 3 histologic variants of the tumor and defined the features of each [32]. Their observations were prompted by earlier studies that Kint and Verlinden had conducted 13 years earlier [40].

Type 1 acquired digital fibrokeratoma was the most common variant observed in 78\% (39 of 50) of Kint et al.'s [32] cases. The features corresponded to those described by Bart et al. [33]. It is mainly a dome-shaped papule that contains thick, dense and closely packed collagen bundles (with fibroblasts between the bundles) that are either irregularly arranged or oriented in the vertical axis of the lesion; fine elastic fibers and numerous capillaries are present [32].

In contrast type II acquired digital fibrokeratoma is mainly tall and hyperkeratotic. It was only observed in 16\% (8 of 50) Kint et al.'s cases [32]. Similar to type I acquired digital fibrokeratoma, in type II acquired digital fibrokeratoma the thick and closely packed collagen bundles are oriented along the main vertical axis of the tumor and numerous capillaries are present; however, compared to type I acquired digital fibrokeratoma, increased fibroblasts (grouped in bundles) and decreased elastic fibers are present. The pathologic features on hematoxylin and eosin staining of type II acquired digital fibrokeratoma has prompted several investigators to consider the lesion to have characteristics suggestive of either superficial acral fibromyxoma or cellular digital fibroma [8, 11, 34].

Type III acquired digital fibrokeratoma was flat to dome-shaped. It was the least common variant of Kint et al.'s [32] cases and was only observed in $6 \%$ (3 of 50) of individuals. The collagen bundles were few, very thin and irregularly arranged. The dermal stroma was poorly cellular and edematous with no elastic fibers [32].

Similar to cellular digital fibroma (yet in contrast to superficial acral fibromyxoma), the acquired digital fibrokeratoma do not have a myxoid stroma and therefore typically do not show positive staining of the dermal stroma with alcian blue stain or periodic acid-Schiff stain [32]. However, in contrast to both superficial acral fibromyxoma and cellular digital fibroma which show diffuse and strongly positive CD34 staining, acquired digital fibrokeratoma is only focally positive or negative after staining with CD34 [3]. To the best of our knowledge, the results of CD99 staining of acquired digital fibrokeratoma have not been described; although Fetsch et al. may have performed CD99 staining on the acquired digital fibrokeratomas they evaluated, the results were not reported in their paper [11].

\section{Work-Up}

A cellular digital fibroma is a benign vascular lesion. It is solitary. However, some of the 
lesions-especially if they involve the nail fold-may be difficult to differentiate clinically from a superficial acral fibromyxoma. Since superficial acral fibromyxoma can invade bone, either a roentgenogram or an ultrasound or a magnetic resonance imaging of the affected digit may be performed prior to biopsy [41]. A 27-year-old man with a slightly painful cellular digital fibroma on the proximal nail fold of his right second toe had a negative roentgenogram of the affected digit [1].

Microscopic evaluation of the tumor is necessary to establish the diagnosis. Therefore, either an excisional biopsy (which provides the entire lesion to be studied and ensures that it has been completely removed) or a biopsy (performed using a punch or shave technique) that provides adequate tissue for evaluation is necessary.

\section{Pathogenesis}

The pathogenesis of cellular digital fibroma remains to be established. Trauma has been observed in patients with superficial acral fibromyxoma [11, 12]. However, none of the patients with cellular digital fibroma have had a prior history of trauma to the lesion site.

Some investigators have suggested that cellular digital fibroma is a variant of superficial acral fibromyxoma $[7,10,36]$. In response, other researchers-who consider cellular digital fibroma to be a distinct entity-replied that the cases of cellular digital fibroma that they reported would then need to be called 'nonmyxoid fibromyxomas' [8]. Subsequently, other authors have referred to cellular digital fibroma as either a myxoid-poor fibroblast-rich superficial acral fibromyxoma or a mucin-poor variant of superficial acral fibromyxoma [9].

Fetsch et al.-who described superficial acral fibromyxoma-commented that they could not rule out the possibility that the type II variant of acquired digital fibrokeratoma was related to superficial acral fibromyxoma [11]; indeed, although they lacked proof, the researchers proposed that some of the type II acquired digital fibrokeratomas were indeed early stage superficial acral fibromyxoma [11]. However, they were confident that the acquired digital fibrokeratoma they studied were significantly different from the superficial acral fibromyxoma they were describing. Specifically, the acquired digital fibrokeratoma had minimal myxoid stroma, were less vascular and were more superficial [11].

McNiff et al. subsequently commented [8], based on the features of the type II variant of the acquired digital fibrokeratoma described by Fetsch et al. [11], that Fetsch et al.'s cases of acquired digital fibrokeratoma may be closely related to those that they had reported as cellular digital fibroma [3]. Hence, it might be reasonable to hypothesize that the shared features of cellular digital fibroma, superficial acral fibromyxoma and acquired digital fibrokeratoma is not merely coincidence but suggestive that the 3 tumors have related genomics yet different phenotypic expression [34].

\section{Treatment}

The management of cellular digital fibroma has been reported for some of the patients. Only 1 of the 14 patients described by McNiff et al. [3] - a 67-year-old man with a tumor on his right third finger-had a re-excision of the cellular digital fibroma since the investigators were concerned about the possibility that the lesion might represent a dermatofibrosarcoma protuberans. The subsequent management of the other 13 patients was not provided [3].

The residual cellular digital fibroma from 2 men, following biopsy, was excised. The cellular digital fibroma on the right index finger of a 38 -year-old man was completely removed and there was no recurrence during the next 18 months of follow-up [2]. Also, the residual tumor on the right second toe of a 27-year-old man was excised [1].

The patient described in this paper-the 61-year-old man with a cellular digital fibroma on the lateral side of his left great toe-had a biopsy that consisted of an elliptical excision. Clinically, there was normal-appearing skin at the periphery of the tissue specimen; a narrow tumor-free surgical margin was confirmed on pathology evaluation. Polypropylene (prolene) 
suture was used to close the biopsy wound (Fig. 3).

The sutures were removed at his follow-up visit 2 weeks. Examination 1 week later showed superficial desquamation of the skin surrounding the excision site (Fig. 4). He did not experience any recurrence over 5 months of followup.

In summary, the management of cellular digital fibroma has only been described in $20 \%$ (4 of 20) of the patients. Three of the men, in whom tumor was still present following the initial biopsy, had the residual lesion completely excised [1-3]. The fourth man, reported in this paper, had an excisional biopsy that completely removed his cellular digital fibroma.

\section{Recurrence}

Information from follow-up examination was provided for only 6 of 20 patients with cellular digital fibroma; none of the patients had a recurrence of their cellular digital fibroma. Two of 14 from McNiff et al. [3] did not have recurrence at follow-up examination. However, the duration of follow-up was not provided; in addition, the researchers did not describe how many of the tumors were present at the margins of the biopsy specimen they evaluated [3].

The cellular digital fibroma-free follow-up of the other 4 men ranged from 5 to 18 months (median, 18 months, mean, 15 months). Two of men had their cellular digital fibroma completely removed either at biopsy (in our patient, a 61-year-old man, described in this paper with a lesion on his left great toe) or during a subsequent excision of residual tumor following biopsy (in a 38-year-old man with a lesion on his right index finger) [2]; recurrence was not observed on examination 5 months and 18 months after the excisional biopsy or re-excision, respectively. Whether the tumor was removed at biopsy was not described for a 55-year-old man with a cellular digital fibroma on his right index finger; however, there was no recurrence when he was evaluated 18 months after the biopsy had been performed [5]. The fourth patient, a 60 -year-old man with a cellular digital fibroma on his right index finger, had residual tumor at the margins of his biopsy specimen; although the site of the residual cellular digital fibroma was not excised, there was no recurrence of the tumor at his evaluation 18 months later [6].

\section{CONCLUSION}

Cellular digital fibroma is a benign fibrous tumor of acral fingers and toes. To date, cellular digital fibroma has been described in 20 patients; however, the actual number of cellular digital fibroma may be higher since the tumor may be mistaken as a superficial acral fibromyxoma or acquired digital fibrokeratoma morphologically by the clinician or microscopically by either the pathologist or both. Cellular digital fibroma has been observed in 12 men and 8 women. The diagnosis age varied from the third decade to the ninth decade; the median diagnosis age was 52 years. The tumor is usually asymptomatic and grows slowly; there is no prior history of trauma at the tumor site. It is often located on either the dorsal, lateral or ventral side of a digit; however, some of the lesions have involved the digit's nail fold. The known incidence of ectopic cellular digital fibroma-in which the tumor was not located on a digit-is $10 \%$; this includes 2 of the 20 patients with lesions on either the palm or the dorsal foot. A cellular digital fibroma is small; for most of the tumors the greatest measurement was only $5 \mathrm{~mm}$. It typically presents as either an erythematous or flesh-colored, solitary papule. Microscopic features include hyperkeratosis and acanthosis of the epidermis, occasionally with a collarette of epithelium extending into the dermis and surrounding the tumor. Within the papillary and upper reticular dermis there is a prominent cellular proliferation of spindle-shaped fibroblasts that do not show atypia or mitoses; there may be a slight myxoid component in the dense collagenous dermal stroma. Cellular digital fibroma does not extend beyond the mid reticular dermis; therefore, there is no erosion of the digital phalanx bone by the tumor. The immunoperoxidase staining profile of cellular digital fibroma shows diffuse and strongly positive CD34 staining 
throughout the entire tumor, positive vimentin staining, and focally positive staining cells for Factor XIIIa. CD99 has only been evaluated in two tumors; one tumor showed focal positive staining. Cellular digital fibroma does not stain positive with EMA or S100; also, individual tumors have not shown positive staining with desmin, muscle-specific actin, smooth muscle actin and tryptase. The clinical and pathologic differential diagnosis of cellular digital fibroma includes other acral tumors such as superficial acral fibromyxoma and acquired digital fibrokeratoma. Similar to cellular digital fibroma, superficial acral fibromyxoma shows diffuse strongly positive staining with CD34. In contrast to cellular digital fibroma, acquired digital fibrokeratoma is CD34-negative or may show focal CD34 positive staining. The pathogenesis of cellular digital fibroma remains to be established. The treatment of cellular digital fibroma is conservative complete excision; however, recurrence has not been observed-even for tumors that have only been partially removed. Although some researches may consider cellular digital fibroma, superficial acral fibromyxoma and acquired digital fibrokeratoma to be variants of the same tumor, the morphologic features, lesion behavior and pathology findings support categorizing them as separate entities.

\section{ACKNOWLEDGEMENTS}

We thank the participant of the study.

Funding. No funding or sponsorship was received for publication of this article.

Authorship. All named authors meet the International Committee of Medical Journal Editors (ICMJE) criteria for authorship of this manuscript, take responsibility for the integrity of the work as a whole, and have given final approval of the version to be published. The opinions expressed in the manuscript are those of the authors. No funding was received for publication of this article.
Disclosures. Robert S. Alpert and Antoanella Calame have nothing to disclose. Philip R. Cohen is a member of the journal's Editorial Board.

Compliance with Ethics Guidelines. This article is based on previously conducted studies and does not contain studies with human participants or animals performed by any of the authors. However, the patient signed a consent providing permission for including the clinical photographs in this article.

Data Availability. Data sharing is not applicable to this article as no data sets were generated or analyzed during the current study.

Open Access. This article is licensed under a Creative Commons Attribution-NonCommercial 4.0 International License, which permits any non-commercial use, sharing, adaptation, distribution and reproduction in any medium or format, as long as you give appropriate credit to the original author(s) and the source, provide a link to the Creative Commons licence, and indicate if changes were made. The images or other third party material in this article are included in the article's Creative Commons licence, unless indicated otherwise in a credit line to the material. If material is not included in the article's Creative Commons licence and your intended use is not permitted by statutory regulation or exceeds the permitted use, you will need to obtain permission directly from the copyright holder. To view a copy of this licence, visit http://creativecommons.org/licenses/bync/4.0/.

\section{REFERENCES}

1. Fernandez Bussy R, Estrella V, Seletti M, Gorosito M, Fernandez Bussy RA. Cellular digital fibroma: distinctive CD34 positive tumor. Revista Argentina De Dermatologia. 2013;94(3):Article 7. http://radonline.org.ar/2013/10/01/fibroma-digital-celularun-tumor-periungueal-cd34-positivo/. Accessed 27 June 2020. 
2. Lee JY, Park SE, Shin SJ, Kim CW, Kim SS. Diagnostic pitfalls of differentiating cellular digital fibroma from superficial acral fibromyxoma. Ann Dermatol. 2015;27(4):462-4.

3. Mc Niff JM, Subtil A, Cowper SE, Lazova R, Glusac EJ. Cellular digital fibromas: distinctive CD34-positive lesions that may mimic dermatofibrosarcoma protuberans. J Cutan Pathol. 2005;32(6):413-8.

4. Pitha J, Smoller BR, Somach S, McCalmont TH. A spindled cell CD34 + dermal proliferation. Am J Dermatopathol. 2002;24(1):85-8.

5. Marin-Cabanas I, Frances L, Bouret A, Gonzalez I, Hispan P, Niveiro $M$, Blanes $M$, Banuls J. CD34 + cellular digital fibroma, about a case. ISSUU GEDP 2014 Malaga. 2014. https://issuu.com/ gedp_alicante/docs/

posterfibromadigitalcelularcd34. Accessed 27 June 2020.

6. Yang H, Yu L. Cutaneous and superficial soft tissue CD34 + spindle cell proliferation. Arch Pathol Lab Med. 2017;141(8):1092-100.

7. Guitart J, Ramirez J, Laskin WB. Cellular digital fibromas: what about superficial acral fibromyxoma? J Cutan Pathol. 2006;33(11):762-3.

8. McNiff JM, Subtil A, Cowper SE, Lazova R, Glusac EJ. Reply (to Cellular digital fibromas: what about superficial acral fibromyxoma?). J Cutan Pathol. 2006;33(11):764.

9. Mancuso CJ, Magro CM, Lipner SR. Acquired digital fibrokeratoma presenting as a painless nodule on the right fifth fingernail. Cutis. 2019;103(6):340-2.

10. Prescott RJ, Husain EA, Abdellaoui A, Al-Mahmoud RMW, Khan M, Salman WD, Twaij Z, Zelger BG, Zelger B, Al-Daraji WI. Superficial acral fibromyxoma: a clinicopathological study of new 41 cases from the U.K.: should myxoma (NOS) and fibroma (NOS) continue as part of 21st-century reporting? Br J Dermatol. 2008;159(6):1315-21.

11. Fetsch JF, Laskin WB, Miettinen M. Superficial acral fibromyxoma: a clinicopathologic and immunohistochemical analysis of 37 cases of a distinctive soft tissue tumor with a predilection for the fingers and toes. Hum Pathol. 2001;32(7):704-14.

12. Hollmann TJ, Bovee JVMG, Fletcher CDM. Digital fibromyxoma (superficial acral fibromyxoma): a detailed characterization of 124 cases. Am J Surg Pathol. 2012;36(6):789-98.

13. Cohen PR, Skupsky H, Erickson C, Calame A. Foreign body (solder) and reaction to the foreign body presenting as a cutaneous tender tumor: case report and a new acronym to aid in recalling the differential diagnosis of painful skin lesions. Cureus. 2020;12(2):e6955.

14. Cohen PR, Erickson C, Calame A. Painful tumors of the skin: "CALM HOG FLED PEN AND GETS BACK". Clin Cosmet Investig Dermatol. 2019;12: 123-32.

15. Cohen PR, Rapini RP, Farhood AI. Expression of the human hematopoietic progenitor cell antigen CD34 in vascular and spindle cell tumors. J Cutan Pathol. 1993;20(1):15-20.

16. Cohen PR, Rapini RP, Farhood AI. Dermatopathologic advances in clinical research. The expression of antibody to CD34 in mucocutaneous lesions. Dermatol Clin. 1997;15(1):159-76.

17. Lisovsky M, Hoang MP, Dresser KA, Kapur P, Bhawan J, Mahalingam M. Apolipoprotein D in CD34positive and CD34-negative cutaneous neoplasms: a useful marker in differentiating superficial acral fibromyxoma from dermatofibrosarcoma protuberans. Mod Pathol. 2008;21(1):31-8.

18. Pasello M, Manara MC, Scotlandi K. CD99 at the crossroads of physiology and pathology. J Cell Commun Signal. 2018;12(1):55-68.

19. Kidd ME, Shumaker DK, Ridge KM. The role of vimentin intermediate filaments in the progression of lung cancer. Am J Respir Cell Mol Biol. 2014;50(1):1-6.

20. Cerio R, Griffiths CE, Cooper KD, Nickoloff BJ, Headington JT. Characterization of factor XIIIa positive dermal dendritic cells in normal and inflamed skin. Br J Dermatol. 1989;121(4):421-31.

21. Cohen PR, Rapini RP, Farhood AI. Dermatofibroma and dermatofibrosarcoma protuberans: differential expression of CD34 and factor XIIIa. Am J Dermatopathol. 1994;16(5):573-4.

22. Cohen PR, Rapini RP, Farhood AI. CD34 expression in neural and fibrohistiocytic lesions. Am J Surg Pathol. 1995;19(1):115-6.

23. Pinkus GS, Kurtin PJ. Epithelial membrane antigen-a diagnostic discriminant in surgical pathology: immunohistochemical profile in epithelial, mesenchymal, and hematopoietic neoplasms using paraffin sections and monoclonal antibodies. Hum Pathol. 1985;16(9):929-40.

24. Sedaghat F, Notopoulos A. S100 protein family and its application in clinical practice. Hippokratia. 2008;12(4):198-204.

25. Kahn HJ, Baumal R, Marks A. The value of immunohistochemical studies using antibody to 
S100 protein in dermatopathology. Int J Dermatol. 1984;23(1):38-44.

26. Crepaldi BE, Soares RD, Silveira FD, Taira RI, Hirakawa CK, Matsumoto MH. Superficial acral fibromyxoma: literature review. Rev Bras Ortop (São Paulo). 2019;54(5):491-6.

27. Schwager ZA, Mannava KA, Mannava S, Telang GH, Robinson-Bostom L, Jellinek NJ. Superficial acral fibromyxoma and other slow-growing tumors in acral areas. Cutis. 2015;95(2):E15-9.

28. Ashby-Richardson H, Rogers GS, Stadecker MJ. Superficial acral fibromyxoma: an overview. Arch Pathol Lab Med. 2011;135(8):1064-6.

29. Shih S, Khachemoune A. Acquired digital fibrokeratoma: review of its clinical and dermoscopic features and differential diagnosis. Int J Dermatol. $2019 ; 58(2): 151-8$.

30. Ali M, Mbah CA, Alwadiya A, Nur MM, Sunderamoorthy D. Giant fibrokeratoma, a rare soft tissue tumor presenting like an accessory digit, a case report and review of literature. Int J Surg Case Rep. 2015;10:187-90.

31. Choi JH, Jung SY, Chun JS, Seo JK, Lee D, Hwang SW, Sung HS. Giant acquired digital fibrokeratoma occurring on the left great toe. Ann Dermatol. 2011;23(1):64-6.

32. Kint A, Baran R, De Keyser H. Acquired (digital) fibrokeratoma. J Am Acad Dermatol. 1985;12(5 Pt $1): 816-21$.

33. Bart RS, Andrade R, Kopf AW, Leider M. Acquired digital fibrokeratomas. Arch Dermatol. 1968;97: 120-9.
34. Plaza JA, Suster S, Prieto VG, Sangueza M. Acquired reactive digital fibroma: a clinicopathologic report of 5 cases of a new entity. J Am Acad Dermatol. 2013;69(4):603-8.

35. Chang P, Meaux T, Pacheco GC. Digital fibromas. Report of three cases. Our Dermatol Online. 2017;8(3):315-17. http://www.odermatol.com/ issue-in-html/2017-3-21-fibromas/. Accessed 27 June 2020 .

36. Al-Daraji W, Miettinen M. Superficial acral fibromyxoma: a clinicopathological analysis of 32 tumors including 4 in the heel. J Cutan Pathol. 2008;35(11):1020-6.

37. Cullen D, Recuero JLD, Culle R, Peralto JLR, Kutzner $\mathrm{H}$, Requena L. Superficial acral fibromyxoma: report of 13 cases with new immunohistochemical findings. Am J Dermatopathol. 2017;39(1):14-22.

38. Pinkus H. Discussion-acquired digital fibrokeratomas. Arch Dermatol. 1968;97:128-9.

39. Stingeni L, Covarelli P, Simonetti S, Tomassini GM, Tramontana M, Tomassini MA, Bianchi L. Superficial acral fibromyxoma with CD10 expression: an under recognized featunder recognizedure. G Ital Dermatol Venereol. 2017;152(4):404-6.

40. Kint A, Verlinden L. Acquired digital fibrokeratoma. Arch Belg Dermatol Syphiligr. 1972;28(4):387-94.

41. Lee S, Reid MAR. Superficial acral fibromyxoma: a case report with radiological review. Skeletal Radiol. 2018;47(7):1021-8. 